\title{
Cáncer de próstata, el problema del diagnóstico ¿Es la resonancia multiparamétrica de próstata la solución?
}

\author{
Patricia Guzm6n F'., Antonella Messina².
}

1.Hospital San Juan de Dios. Santiago, Chile.

2. Istituto Nazionale dei Tumori Milano. Milano, Italia.

Prostate cancer, the problem of diagnosis. Is multiparametric prostate magnetic resonance imaging the solution?

Resumen: Junto con un aumento sostenido en la incidencia de cáncer de próstata en los países desarroIlados ha habido un cambio en el enfrentamiento diagnóstico en estos pacientes. El diagnóstico tradicional basado en la medición del antígeno prostático específico y la biopsia randomizada ha mostrado tener muchas falencias ya que sobre-diagnostica cánceres no significativos y sub-diagnostica cánceres clínicamente significativos. La resonancia magnética de próstata multiparamétrica ha demostrado ser útil ya que disminuye este tipo de falencias. En este artículo se revisará la historia de la resonancia magnética de próstata y del PI-RADS, con el objeto de revisar el rendimiento de estos métodos y sus indicaciones actuales.

Palabras clave: Antígeno prostático específico, Cáncer de próstata, Resonancia multiparamétrica de próstata, Vigilancia activa cáncer de próstata.

Abstract: Along with a sustained increase in the incidence of prostate cancer in developed countries, there has been a change in the diagnostic approach in these patients. The traditional diagnosis based on the measurement of the specific prostate antigen and randomized biopsy has shown to have many shortcomings as it leads to overdiagnosis of non-significant cancers and underdiagnosis of clinically significant cancers. The multiparametric prostate magnetic resonance imaging has proven to be useful since it diminishes these shortcomings. In this article we will review part of the history of prostate magnetic resonance imaging and PI-RADS, in order to review the performance of these methods and their current indications. Keyword: Active surveillance prostate, Mesh terms, Multiparametric MRI, Prostate cancer, Prostate-specific Antigen.

Guzmán $P$, et al. Cáncer de próstata, el problema del diagnóstico ¿Es la resonancia multiparamétrica de próstata la solución? Rev Chil Radiol 2019; 25(2): 60-66.

*Correo electrónico: Patricia Guzmán / patyguzfalcon@gmail.com

Trabajo enviado el 24 de abril de 2019. Aceptado para publicación el 21 de junio de 2019.

\section{Introducción}

El cáncer de próstata viene mostrando un aumento sostenido en su incidencia en los países desarrollados, llegando el 2018 a ser el segundo cáncer más frecuente en los hombres luego del cáncer pulmonar en el mundo, ocupando el quinto lugar en mortalidad $^{(1)}$.

Hace pocos años el diagnóstico del cáncer de próstata estaba basado en el tamizaje con antígeno prostático específico (APE), el que se ha usado y se usa masivamente hasta ahora. Por otro lado, la biopsia randomizada es el método utilizado para acceder a muestras para estudio histológico. Esto ha llevado a un sobre-diagnóstico y sobre-tratamiento de lesiones no clínicamente significativas (CNS), es decir, que 
nunca llegarán a producir metástasis y no pondrán en riesgo la vida del paciente.

El sobre tratamiento de dichos cánceres indolentes puede además generar un detrimento en la calidad de vida debido a disfunciones intestinales, urinarias y eréctiles. Con la finalidad de evitar el sobre-tratamiento, estos pacientes comenzaron a seguirse en protocolos de vigilancia activa, sin embargo, algunos de ellos además presentaban lesiones clínicamente significativas (CS) ocultas en lugares de menor alcance para la biopsia randomizada, como la región anterior de la próstata - aquella localizada anteriormente a la uretra - y el ápex prostático y, por lo tanto, mostraban una evolución natural desfavorable, incluso con la aparición de metástasis. Vale recordar que se consideran como cánceres CS todos aquellos con un puntaje de Gleason de 7 hacia arriba.

Es así como se hizo necesario otro método diagnóstico que tuviera la capacidad de diagnosticar solo las lesiones CS.

El uso de la resonancia magnética $(\mathrm{RM})$ de próstata se remonta a la década de los 80 , pero su principal desarrollo se inició alrededor del año 2007, cuando comenzaron a aparecer artículos con el uso de difusión en el estudio de próstata ${ }^{(2,3)}$. La espectroscopia había comenzado a desarrollarse un poco antes. En 2011 la Sociedad Europea de Radiología Urogenital (ESUR) propuso el "Prostate Imaging-Reporting and Data System version 1" (Pi-RADS v1). En 2014 se da a conocer el "Prostate Imaging-Reporting and Data System version 2" (PI-RADS v2) $)^{(4)}$, diseñado en conjunto por el Colegio Americano de Radiología, la ESUR y la Fundación Ad MeTech, con el objetivo de mejorar la detección y caracterización de los cánceres de próstata a través de la RM, sistematizar la técnica de la RM multiparamétrica (RM-mp) de próstata y también simplificar y sistematizar el informe de dichos exámenes.

Luego de la publicación del PI-RADS 2 se han publicado múltiples estudios y se identificaron algunas limitaciones y ambigüedades que se intentan resolver con la versión 2.1 publicada recientemente en marzo del presente $a \tilde{n} 0^{(5)}$.

\section{Técnica de RM-mp de próstata}

La RM-mp de próstata requiere secuencias anatómicas de alta resolución ponderadas en T2, además de secuencias funcionales de difusión (DWI) y las secuencias dinámicas contrastadas (DCE). Las secuencias ponderadas en T1 además se agregan para pesquisar contenido hemático, metástasis nodales y óseas. En el PI-RADS v2 se propone una secuencia considerada dominante para la zona periférica (secuencia de DWI) y una secuencia dominante para la zona de transición (secuencia ponderada en T2).

EI PI-RADS v2 además detalla los requerimientos técnicos mínimos necesarios para una adecuada
RM-mp. En un artículo del $2018^{(6)}$ Steven y otros estudiaron la adherencia de 107 establecimientos a los parámetros técnicos del PI-RADS v2, demostrando un amplio rango de adherencia de entre 20 a 100\% para parámetros individuales de adquisición, concluyendo que es necesaria una difusión aún mayor de estos estándares mínimos. No es el objetivo de este texto individualizarlos, pero se discutirán algunos puntos de las secuencias funcionales:

\section{Secuencia de Difusión}

Para que se obtenga una clara diferenciación entre el tejido no neoplásico y neoplásico se requiere al menos de un valor de b alto de difusión, que en el PI-RADS v2 se estableció como mínimo de 1.400. Un estudio retrospectivo realizado en 2016 por Rosenkrantz y otros ${ }^{(7)}$ demostró que los valores de b entre $1.500 \mathrm{y}$ 2.500 son los óptimos para la detección del cáncer, ya que con estos valores se logra diferenciar las lesiones inflamatorias o fibróticas, que se "apagan con valores de b altos", de aquellas lesiones neoplásicas.

Dado que se requieren valores de $b$ muy altos surge el problema de la poca relación señal ruido (SNR) en estas secuencias y es por esta razón que para realizar una buena RM-mp de próstata se requieren equipos con una inducción magnética de 3 Tesla o de 1,5 Tesla, en estos últimos idealmente con bobina endorectal lo que mejora la relación señal ruido (SNR).

En la última versión del PI-RADS 2.1 se recomienda realizar adquisiciones con un set de b bajo $(0-100$ $\left.\mathrm{sec} / \mathrm{mm}^{2}\right)$ y uno intermedio $\left(800-1000 \mathrm{sec} / \mathrm{mm}^{2}\right)$, con los que se calcula el Mapa ADC. Además, se debe adquirir o calcular un b alto, (mayor a $1.400 \mathrm{seg} / \mathrm{mm}^{2}$ ), este valor se excluye del cálculo del mapa ADC con el propósito de evitar el efecto Kurtosis.

\section{La secuencia dinámica contrastada (DCE)}

La utilidad de la secuencia dinámica se limita a la zona periférica. Cuando es positiva las lesiones dudosas (PI-RADS 3) en DWI son recategorizadas como PI-RADS 4. En la práctica clínica, sin embargo, también se le considera como una secuencia de "back-up" o respaldo, especialmente cuando la difusión se ve degradada por artefactos o presenta una SNR sub-óptima(5).

Algunos trabajos ya han demostrado y cuantificado la utilidad de la secuencia contrastada ${ }^{(8,9,10)}$. El artículo de Taghipour et al(10), que compara resultados de RM-mp versus hallazgos histológicos de prostatectomía radical (PR), mostró la utilidad de la DCE en un $21,5 \%$ de los casos y en estos comprobó que incrementa correctamente el PI-RADS de 3 a 4 en un $68,9 \%$. El trabajo de Visschere et al ${ }^{(9)}$, mostró similares resultados a pesar de tener diferencias metodológicas.

Existen autores que proponen realizar estudios 
bi-paramétricos, solo con secuencias T2 y DWI, por el aumento del tiempo de adquisición, evidencia de depósito tisular de gadolinio y los costos asociados de la utilización de contraste basado en gadolinio, pero debe tenerse en cuenta su utilidad en un porcentaje no despreciable de lesiones. En relación con este punto, la versión 2.1 de PI-RADS da las siguientes recomendaciones:

1. Preferir la RM-mp en hombres con biopsias previa negativas que presenten aumento de APE y aquellos en vigilancia activa que se están evaluando por un rápido tiempo de doblaje del APE o que cambien su status clínico-patológico.

2. Preferir la RM-mp en hombres que previamente se han realizado una resonancia bi-paramétrica sin hallazgos y en los que persiste la sospecha de lesiones CS.

3. En casos de intervenciones previas, ya sean resecciones transrectales, resecciones transuretrales, radioterapia o en aquellos con hormonoterapia.

4. En casos de pacientes que no se hayan realizado biopsia prostática previa pero que presenten antecedentes familiares, factores predisponentes genéticos conocidos, puntaje genómico urinario elevado y puntaje de riesgo mayor al promedio.

5. Hombres con implantes de cadera, en los que probablemente la imagen en difusión se verá degradada.

\section{El paradigma de la secuencia dominante}

El paradigma de la secuencia dominante fue validado en 2017 en un trabajo prospectivo realizado por Greer et al ${ }^{(8)}$, en que participaron 9 radiólogos de todo el mundo. Se revisaron las características en RM-mp de 654 lesiones y su correlación con los hallazgos anatomopatológicos de la prostatectomía radical. En este trabajo se demostró un mejor desempeño de la secuencia ponderada en T2 en la zona de transición que en la periférica, en cambio en la zona periférica la secuencia de DWI supera en desempeño a la secuencia T2. La DCE mostró utilidad en la zona periférica y demostró que su positividad aumenta la probabilidad de cáncer. En este trabajo además se calculó la probabilidad de cáncer de cada categoría de PI-RADS (Tabla 1):

Cabe destacar el porcentaje no despreciable $(15,7 \%)$ de probabilidad de lesiones CS en aquellas catalogadas como PI RADS 2.

\section{La interpretación de los hallazgos y el informe}

La correcta interpretación de los hallazgos de la $\mathrm{RM}-\mathrm{mp}$ requiere un alto grado de experiencia. En un consenso realizado el $2013^{(11)}$ se acordó que los radiólogos que informan RM de próstata deben informar al
Tabla 1. Correlación entre la categoría PI-RADS en la primera columna con cánceres clínicamente significativos en la segunda columna y con la totalidad de cánceres en la tercera, según trabajo de Greer et al.

\begin{tabular}{|l|c|c|}
\hline & $\begin{array}{c}\text { Cánceres } \\
\text { CS (\%) }\end{array}$ & $\begin{array}{c}\text { Cánceres } \\
\text { CS + no CS (\%) }\end{array}$ \\
\hline Pirads 2 & 15,7 & 24,3 \\
Pirads 3 & 33 & 40 \\
Pirads 4 & 70 & 78,7 \\
Pirads 5 & 90,7 & 91,3 \\
\hline
\end{tabular}

menos 50 exámenes al año y asistir frecuentemente a reuniones de correlación anatomo patológicas, ya que se considera que se requiere una gran destreza de interpretación, algo similar a lo que ocurre en la interpretación de la mamografía.

\section{Rendimiento del PI-RADS V2}

Varios son los estudios que han verificado el rendimiento de la segunda versión del PI-RADS. Un metaanálisis del $2017^{(12)}$ analizó 13 estudios publicados hasta la fecha, todos ellos retrospectivos, y compararon los hallazgos de RM con la anatomía patológica de biopsia de próstata guiada con ultrasonido o por RM, o bien con los hallazgos de prostatectomía radical. En este metaanálisis se observó una sensibilidad general de $85 \%$ y una especificidad general de $71 \%$. Los valores predictivos positivos (VPP) variaron entre $54 \%$ a $97 \%$ y los valores predictivos negativos (VPN) entre $26 \%$ a $92 \%$. Un estudio más reciente, Taghipour et al ${ }^{(10)}$ mostraron una sensibilidad de $89,2 \%$, especificidad de $57,6 \%$, VPP de $88,3 \%$ y VPN de $59,6 \%$ con una exactitud diagnóstica de un $82,3 \%$ en la detección de cánceres clínicamente significativos.

En resumen, los distintos trabajos publicados hasta la fecha muestran una alta sensibilidad del PI-RADS v2 y una moderada especificidad en el diagnóstico de cáncer de próstata CS.

No es el objeto de este artículo hacer una revisión pictórica de las distintas lesiones según el PI-RADS v2, pero las figuras 1 y 2 ejemplifican lesiones $\mathrm{PI}$ RADS 4 y 5, confirmadas con biopsia fusión en las zonas de transición y periférica, respectivamente.

\section{Biopsia "target" prostática}

Una vez detectada una lesión CS en la RM son tres los métodos usados para la biopsia de dichas lesiones "targets":

1. Biopsia cognitiva: En la biopsia cognitiva el médico que realizará la biopsia debe juntar en su cabeza la imagen obtenida en la resonancia 


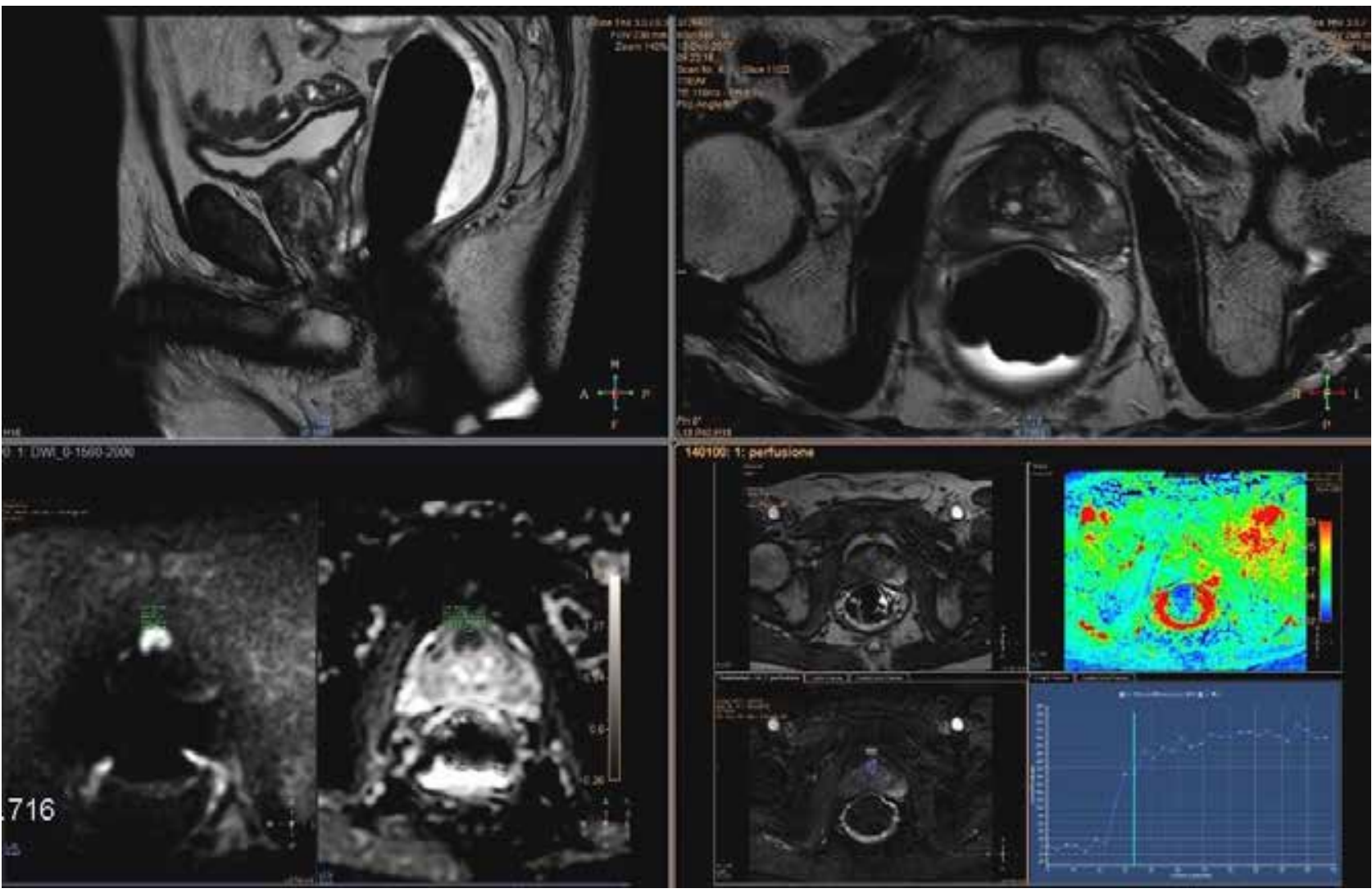

Figura 1: Lesión PI-RADS 4 localizada en la región anterior de la próstata, en la zona de transición. La lesión es hipointensa en T2, muestra importante restricción en secuencia de difusión (b 2000) con correlato en mapa ADC (imagen inferior derecha). En secuencia dinámica contrastada muestra moderado realce con curva contraste-tiempo de tipo 2.

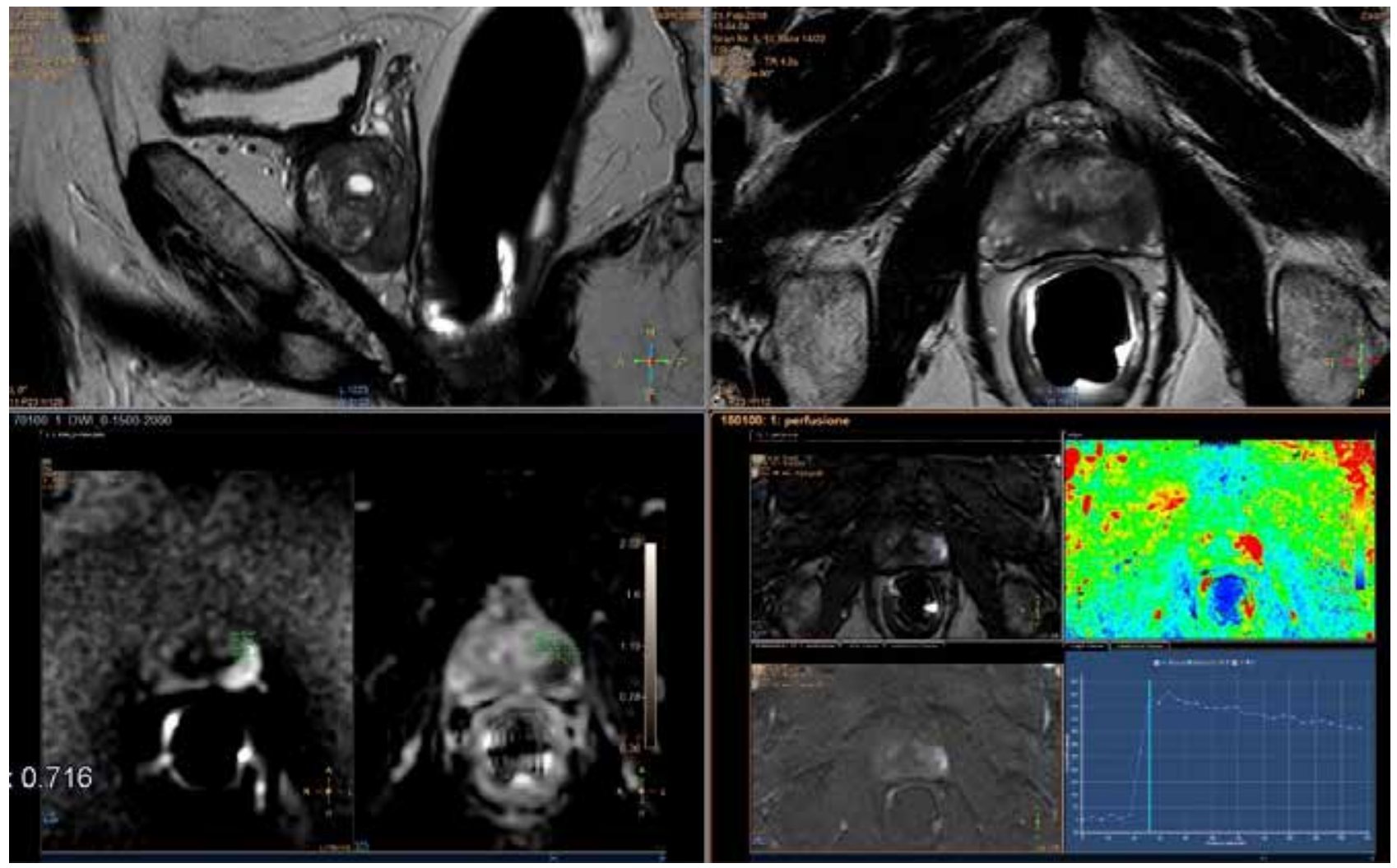

Figura 2: Lesión PI-RADS 5 en la zona periférica izquierda. La lesión es hipointensa en T2. Nótese la restricción en secuencia de difusión (b 2000) con correlato en mapa ADC. La imagen inferior izquierda corresponde a la secuencia dinámica, la lesión muestra gran vascularización, con una curva contraste-tiempo de tipo 3. 
de próstata con aquella que ve en la ecografía transrectal y biopsiar la lesión vista en RM.

2. Biopsia guiada por RM: En este método la biopsia se realiza en el resonador. Se obtienen menos cilindros de biopsia, los que se limitan a la lesión "target", disminuyendo la morbilidad asociada al procedimiento. Se puede realizar via transrectal, transperineal o transglútea. La desventaja es que requiere el aparataje compatible con RM y ocupa tiempo del resonador, por lo que su disponibilidad es muy baja.

3. Biopsia fusión. En este método la imagen obtenida en RM se fusiona con la imagen de la ecografía transrectal y se marcan las lesiones sospechosas, por lo que el operador ve en tiempo real lo que debe biopsiar (Figura 3). En general, además de los "target" se realizan biopsias aleatorias del resto de la próstata, al igual que en la biopsia cognitiva.

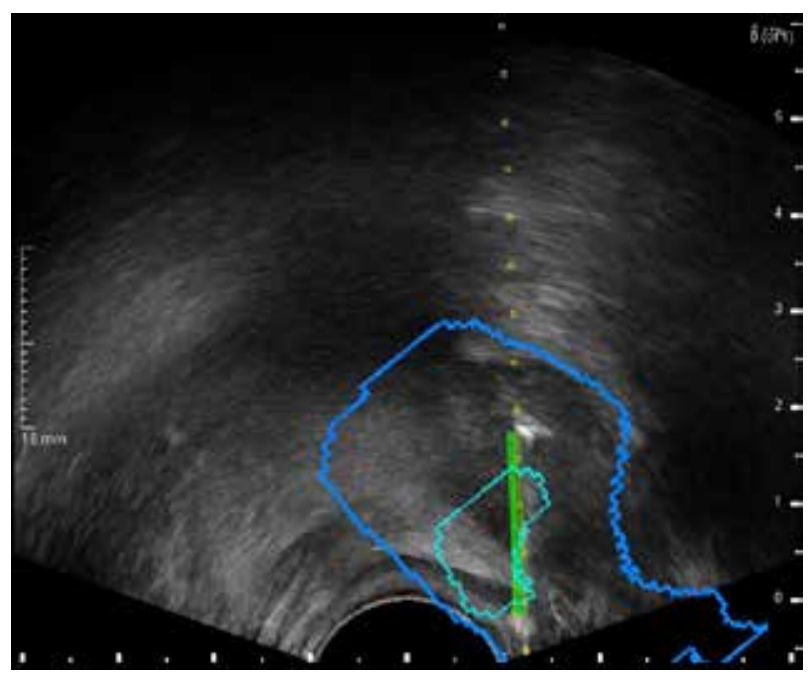

Figura 3: Imagen de biopsia fusión de lesión prostática. La marca en azul corresponde a los límites de la próstata, la línea calipso a la demarcación de la lesión a biopsiar y las líneas verdes corresponden a las marcas de los "disparos" de la biopsia.

Existen varios artículos que comparan los distintos tipos de biopsia de la próstata, mostrando distintos resultados. Uno de ellos comparó la biopsia aleatoria o randomizada, con biopsia cognitiva y con la biopsia fusión. Las tasas de detección de cáncer fueron de $32 \% ; 34,6 \%$ y $48 \%$ respectivamente, demostrando un rendimiento significativamente mejor de la biopsia fusión ${ }^{(13)}$. Un estudio prospectivo randomizado más reciente (ensayo FUTURO), publicado en noviembre del $2018^{(14)}$ estudió el rendimiento de los tres métodos de biopsia "target". En este estudio 234 pacientes fueron asignados aleatoriamente a biopsia cognitiva, biopsia guiada por RM o biopsia fusión. Sorpresivamente, no se vieron diferencias significativas en la tasa de detección de cáncer de próstata entre los tres métodos, las que son de 49,4\% para la biopsia fusión, $43,6 \%$ para la biopsia cognitiva y $54,5 \%$ para la biopsia guiada por RM. Los autores concluyen que el método de elección dependerá del equipamiento con el que se cuenta y la experiencia local de cada establecimiento.

\section{Anatomía Patológica}

La Sociedad Internacional de Patología Urológica ha emitido en el 2014 un nuevo sistema de graduación del cáncer de próstata resultado de un consenso ${ }^{(15)}$. Este nuevo sistema de graduación tiene el beneficio doble de predecir el pronóstico y además facilitar la comunicación con el paciente. Es así como se establecieron cinco clasificaciones, las que se detallan en la tabla 2.

Tabla 2. Equivalencia de la clasificación ISUP en la primera columna con el Score de Gleason en la segunda y la clasificación de riesgo del cáncer de próstata en la última.

\begin{tabular}{|l|c|c|}
\hline ISUP & $\begin{array}{c}\text { SCORE } \\
\text { DE GLEASON }\end{array}$ & RIEGO \\
\hline $\mathbf{1}$ & $<0=6$ & Bajo \\
$\mathbf{2}$ & $3+4$ & Intermedio \\
$\mathbf{3}$ & $4+3$ & Alto \\
$\mathbf{4}$ & 8 & Alto \\
$\mathbf{5}$ & $9-10$ & Alto \\
\hline
\end{tabular}

Es sumamente importante que los radiólogos que interpretan e informan los estudios de RM de próstata estén familiarizados con la terminología actual.

\section{La reinvención del antígeno prostático específico}

Tradicionalmente el APS era la piedra angular en el tamizaje del cáncer de próstata, sin embargo, su baja especificidad ha llevado al sobre diagnóstico y sobre tratamiento del cáncer de próstata. Actualmente el valor de la densidad del APE (valor APE/volumen de la próstata) se considera como mejor indicador de la presencia de una lesión clínicamente significativa, siendo el valor de corte más utilizado de más de 0,15. Otro indicador utilizado es el tiempo de doblaje del APE, considerándose un valor de corte de 3 años y menos. 


\section{Rol de la RM en la vigilancia activa del cáncer de próstata}

El propósito de la vigilancia activa es minimizar la morbilidad del tratamiento del cáncer de próstata preservando la eficacia oncológica ${ }^{(16)}$. Se utiliza en aquellos pacientes en los que se diagnostican lesiones CNS, o de bajo riesgo (en general aquellas con puntaje de Gleason de $3+3$ ). La RM-mp tiene dos grandes indicaciones en este contexto.

1. Ingreso a vigilancia activa: Una vez que el paciente se ha realizado una biopsia randomizada y quiere ser ingresado a un protocolo de vigilancia activa se utiliza la RM-mp para asegurarse que no existan otras lesiones que sean CS.

2. Control evolutivo de lesiones CNS: Aquellas lesiones CNS (diagnosticadas con biopsia randomizada) pueden ser controladas con RM-mp. Un estudio retrospectivo verificó la variación en los hallazgos de RM-mp de próstata en 86 pacientes en vigilancia activa con lesiones CNS, demostrando que un $17 \%$ de aquellos que no tenían lesiones visibles en la primera RM desarrollaban una lesión en un promedio de 3,6 años ${ }^{(17)}$. Los autores concluyen que en estos pacientes sería prudente un control con frecuencia de dos años, lo que evitaría biopsias innecesarias, disminuyendo la morbilidad asociada.

\section{Conclusiones}

El cáncer de próstata es el segundo cáncer que más afecta al hombre en el mundo. Su método de diagnóstico tradicional basado en la medición del APE y biopsia randomizada de próstata adolece de importantes problemas. La RM-mp de próstata, en conjunto con el PI RADS v2, han demostrado tener alta sensibilidad y moderada especificidad en el diagnóstico de lesiones CS cuando se realiza en condiciones técnicas óptimas y son interpretadas por radiólogos capacitados, por lo que actualmente juega un rol preponderante en el diagnóstico de cáncer de próstata. Es así como en las Guías Clínicas Europeas de cáncer de próstata de 2019 ya se aconseja su uso previo a cualquier biopsia prostática ${ }^{(18)}$, por lo que solo se espera un aumento sustantivo de la realización de este tipo de examen en los siguientes años.

\section{Referencias}

1. Bray F., Ferlay J., Soerjomataram I., Siegel R.L., Torre L.A., Jemal A. Global cancer statistics 2018: GLOBOCAN estimates of incidence and mortality worldwide for 36 cancers in 185 countries. CA: A Cancer Journal for Clinicians 2018; 68: 394-424.

2. Haider M.A., Van der Kwast T.H., Tanguay, et al. Combined T2 -weighted and diffusion-weighted MRI for localization of prostate cancer. AJR Am J Roentgenol. 2007 Aug; 189(2): 323-328.
3. Morgan V.A., Kyriazi SI, Ashley S.E., et al. Evaluation of the potential of diffusion-weighted imaging in prostate detection. Acta Radiol. 2007 Jul; 48(6): 695-703.

4. American College of Radiology. PI-RADS V 2: Prostate Imaging Reporting and Data System version 2.0. American College of Radiology. website www.acr.org/ Quality-Safety/Resources/PIRADS. Published 2015. Accessed January 24, 2016.

5. Turkbey B, et al. Prostate Imaging Reporting and Data System Version 2.1: 2019 Update of Prostate Imaging Reporting and Data System Version 2. Eur Urol 2019, https://doi.org/10.1016/j.eururo.2019.02.033.

6. Steven J., Esses MD, Samir S., Taneja, MD, Andrew B., Rosenkrantz MD. Imaging Facilities Adherence to PI-RADS v2 Minimum Technical Standards for the Performance of Prostate MRI. Acad Radiol 2018; 25: 188-195.

7. Rosenkrantz AB, Parikh N, Kierans AS, Kong MX, Babb JS, Taneja S, Ream JM. Prostate Cancer Detection Using Computed Very High b-value Diffusion-weighted Imaging: How High Should We Go? Acad Radiol 2016; 23: 704-711.

8. Greer MD., Shih JH., Lay N, Barrett T, Bittencourt LK, Borofsky S, Kabakus IM., Law YM, Marko J, Shebel H, Mertan FV, Merino MJ, Wood BJ, Pinto PA. , Summers RM, Choyke PL., Turkbey B. et al Validation of the Dominant sequence Paradigm and role of Dynamic contrast-enhanced imaging in Pi-RADS Version 2. Radiology 2017 december; 285(3).

9. De Visschere PI, Lumen N, Ost P, et al. Dynamic contrast- enhanced imaging has limited added value over T2-weighted imaging and diffusion-weighted imaging when using PI-RADSv2 for diagnosis of clinically significant prostate cancer in patients with elevated PSA. Clin Radiol 2017; 72(1): 23-32.

10. Taghipour M, Ziaei A, Alessandrino F, Hassanzadeh $E$, Harisinghani $M$, Vangel $M$, Clare $M$, Tempany $F$, Fennessy M. Investigating the role of DCE-MRI, over T2 and DWI, in accurate PI-RADS v2 assessment of clinically significant peripheral zone prostate lesions as defined at radical prostatectomy. Published online. Abdominal Radiology October 2018.

11. Kirkham AP, Haslam P, Keanie JY, McCafferty I, Padhani AR, Punwani S, Richenberg J, Rottenberg G, Sohaib A, Thompson P, Turnbull LW, Kurban L, Sahdev A, Clements R, Carey BM, Allen C. Prostate MRI: Who, when, and how? Report from a UK consensus meeting. Clin Radiol. 2013 Oct; 68(10): 1016-1023.

12. Zhang L, Tang M, Chen S, Lei $X$, Zhang $X$, Huan $Y$. A meta-analysis of use of prostate imaging reporting and data system version 2 (PI-RADS V2) with multi parametric MR imagine for the detection of prostate cancer. European Radiology. 2017 december; 27(2): 5204-5214.

13. Daniel T., Oberlin DD., Casalin FH., Miller RS., Matulewicz Kent T., Perry RB., Nadler SK, William J. Catalona JJ. Meeks. Diagnostic Value of Guided Biopsies: Fusion and Cognitive-registration Magnetic Resonance Imaging Versus Conventional Ultrasound Biopsy of the Prostate. UROLOGY 2016; 92: 75-79.

14. Wegelin O, et al. The FUTURE Trial: A Multicenter Randomised Controlled Trial on Target Biopsy Techniques Based on Magnetic Resonance Imaging in the 
Diagnosis of Prostate Cancer in Patients with Prior Negative Biopsies. Eur Urol 2018.

15. Egevad L, Delahunt B, Srigley JR, Samaratunga $H$. International Society of Urological Pathology (ISUP) grading of prostate cancer - An ISUP consensus on contemporary grading. Journal of Pathology, Microbiology and Immunology. 2016 jun; 124(6): 433-435.

16. Parker C. Active surveillance: towards a new paradigm in the management of early prostate cancer. Lancet Oncol. 2004; 5: 101-106.

17. Giganti F, Moore CM., Punwani S, Allen C, Emberton $M$, Kirkham A. The natural history of prostate cancer on $\mathrm{MRI}$ : lessons from an active surveillance cohort. Prostate Cancer and Prostatic Diseases 2018; 21: 556-563.

18. EAU Guidelines. Edn. presented at the EAU Annual Congress Barcelona 2019. ISBN 978-94-92671-04-2. 